\title{
EFEITO DE TRATAMENTOS TÉRMICOS NAS PROPRIEDADES MECÂNICAS DE CHAPAS SOLDADAS DO AÇO INOXIDÁVEL DUPLEX UNS S32304
}

\author{
Gabriela Aksascki Caetano ' \\ Temístocles de Souza Luz ${ }^{2}$ \\ Lucas Mirandola Coelho ${ }^{2}$ \\ Jesualdo Luiz Rossi ${ }^{3}$ \\ Marcos Tadeu D'Azeredo Orlando '
}

\section{Resumo}

O aço inoxidável duplex UNS S32304 tem sido investigado como candidato para construção de um receptáculo para transporte de material radioativo associado ao futuro Reator Multipropósito Brasileiro (RMB). O foco deste trabalho é avaliar o efeito de diferentes temperaturas de tratamento térmico em chapas de UNS S32304 com espessura de I,8mm após processo de soldagem TIG autógeno. Utilizou-se corrente pulsada com polaridade direta, gás de proteção com uma mistura de argônio e $2 \%$ de nitrogênio e um sistema de acionamento automático com controle digital dos parâmetros. Os corpos de prova soldados e como recebido foram tratados termicamente durante $8 \mathrm{~h}$ a temperaturas de 475,600 e $750^{\circ} \mathrm{C}$ seguido de resfriamento ao ar. As amostras foram submetidas a ensaios de tração com taxa de deformação de $10^{-4} \mathrm{~s}^{-1}$. Comprovou-se que somente a amostra tratada a $600^{\circ} \mathrm{C}$ mostrou um comportamento tensão-deformação similar à amostra como recebida. Para as amostras soldadas, o tratamento a $600^{\circ} \mathrm{C}$ aumentou o limite de resistência à tração e o alongamento \% total em relação à amostra somente soldada e sem tratamento térmico.

Palavras-chave: Aço inoxidável duplex; Tratamento térmico; Tensão-deformação; Soldagem TIG.

\section{EFFECT OF HEAT TREATMENTS ON THE MECHANICAL PROPERTIES IN UNS S32304 DUPLEX STAINLESS STEEL WELDED PLATES}

\begin{abstract}
The duplex stainless steel UNS S32304 has been investigated as a candidate for the construction of a receptacle for the transport of radioactive material associated with the future Brazilian Multipurpose Reactor (RMB). This work was focused on the effect of different heat treatment temperatures in UNS S32304 samples with $1.8 \mathrm{~mm}$ of thickness after autogenous GTAW welding process. Pulsed current was used with direct polarity, argon and $2 \%$ of nitrogen as shielding gas and an automatic drive system with digital control of the parameters. The welded and received specimens were heat treated for $8 \mathrm{~h}$ at 475,600 and $750^{\circ} \mathrm{C}$ and subsequent air cooling. These samples were submitted to tensile tests using deformation rate of $10^{-4} \mathrm{~s}^{-1}$. It was verified that only samples treated at $600^{\circ} \mathrm{C}$ showed a stress-strain behavior similar to samples as received. For the welded samples, the heat treatment at $600^{\circ} \mathrm{C}$ improve the maximum tensile stress and ductility as compared with welded sample without heat treatment.
\end{abstract}

Keywords: Duplex stainless steel; Heat treatment; Stress-strain; GTAW welding.

\section{INTRODUÇÃO}

Os aços inoxidáveis duplex (AID) são compostos por uma estrutura mista de ferrita e austenita, em proporções aproximadamente iguais, o que promove a combinação de excelentes propriedades mecânicas e elevada resistência à corrosão [1-10], e torna estes materiais bastante atrativos do ponto de vista econômico e tecnológico $[1,4,1|-| 3]$. O equilíbrio dessas fases é alcançado através de tratamento térmico de solubilização, seguido de um resfriamento

'Programa de Pós-graduação em Engenharia Mecânica, Universidade Federal do Espírito Santo - UFES, Vitória, ES, Brasil. E-mail: biaksascki@gmail.com ${ }^{2}$ Departamento de Engenharia Mecânica, Universidade Federal do Espírito Santo - UFES, Vitória, ES, Brasil.

${ }^{3}$ Instituto de Pesquisas Energéticas e Nucleares - IPEN, São Paulo, SP, Brasil. 
rápido para evitar a precipitação de outras fases [14]. Essas precipitações ocorrem numa faixa de temperatura de 300 a $1000^{\circ} \mathrm{C}$, como resultado de condições de serviço, ciclos térmicos de soldagem ou tratamentos térmicos, e torna mais propensa a transformação da fase ferrítica em compostos intermetálicos (sigma, chi), nitretos, carbonetos e a fase alfa linha, que podem levar a redução de tenacidade, ductilidade e resistência à corrosão $[1,3,8,1 \mathrm{I}, 14]$.

Os AID têm sido desenvolvidos para terem uma excelente soldabilidade. Uma vez que grande parte das aplicações atuais desses aços envolve algum processo de soldagem $[2,14]$. Contudo, a exposição do material a ciclos térmicos durante o processo de soldagem favorece a precipitação de fases deletérias, modifica a microestrutura e as propriedades do aço $[7,15]$ e leva ao desenvolvimento de tensões residuais na junta soldada, que podem gerar formação de trincas e maior propensão para a ocorrência de fadiga ou fratura frágil [16]. Tratamentos térmicos são realizados para que o material atinja determinadas propriedades mecânicas, que visam à remoção de tensões, aumento da resistência mecânica, melhora da ductilidade, usinabilidade, resistência ao desgaste, entre outros [II].

Objetivando melhorar as propriedades mecânicas pós-soldagem do AID UNS S32304, corpos de prova foram submetidos a diferentes temperaturas de tratamento térmico, e para avaliar os efeitos dos tratamentos térmicos sobre as propriedades mecânicas pós-soldagem do material, foram realizados ensaios de tração em um simulador termomecânico. Ressalta-se que este material é candidato a revestimento externo do receptáculo para transporte de materiais radioativos do Reator Multipropósito Brasileiro (RMB).

\section{METODOLOGIA}

Foram utilizadas tiras de um AID de baixa liga, caracterizado como UNS S32304 com espessura de I,8mm e $72,0 \mathrm{~mm}$ de largura. A composição química foi caracterizada por fluorescência de raios $X$ ( $F R X)$ em um espectroscópio da marca Shimadzu, modelo EDX-720, e está exposta na Tabela I.

A soldagem das chapas foi do tipo união através do procedimento TIG autógeno, com utilização da máquina de solda Digiplus A7 da IMC Soldagem, e um processo automatizado que manteve constantes a velocidade e a tensão de soldagem. Um sistema da SPS - Sistemas e Processos

Tabela I. Composição química do AID UNS S32304 analisada por FRX

\begin{tabular}{cccccc}
\hline$\% \mathrm{Mn}$ & $\% \mathrm{Cr}$ & $\% \mathrm{Ni}$ & $\% \mathrm{Mo}$ & $\% \mathrm{Cu}$ & $\% \mathrm{Fe}$ \\
\hline $\mathrm{I}, 57(2)$ & $22,55(\mathrm{I})$ & $3,47(\mathrm{I})$ & $0,24(2)$ & $0,36(\mathrm{I})$ & $73(\mathrm{I})$ \\
\hline
\end{tabular}

de Soldagem, modelo MCS 2000, permitiu o suporte e a movimentação da tocha, com controle automático de altura do arco (AVC) através do monitoramento da tensão de soldagem, referenciado por valor pré-estabelecido. O controle digital dos parâmetros foi feito por um sistema de aquisição de sinais de soldagem da IMC Soldagem.

Utilizou-se corrente pulsada com polaridade direta, gás de proteção com uma mistura de argônio (Ar) e 2\% de nitrogênio $\left(\mathrm{N}_{2}\right)$ com vazão de $\mathrm{I} \mathrm{L} / \mathrm{min}$ e gás de purga com uma mistura de $\mathrm{Ar}$ e $10 \%$ de $\mathrm{N}_{2}$ com mesma vazão, com o intuito de proteger a poça de fusão contra oxidação e evitar as perdas de $\mathrm{N}_{2}$ na raiz da solda. O eletrodo utilizado foi de tungstênio dopado com $2 \%$ de tório e afiado a $60^{\circ}$. Os demais parâmetros utilizados na soldagem estão expostos na Tabela 2.

Os tratamentos térmicos foram realizados em forno tipo mufla programável, na região de gradiente de $\pm 2^{\circ} \mathrm{C}$, com precisão de $\pm 5^{\circ} \mathrm{C}$, nas temperaturas de $475^{\circ} \mathrm{C}, 600^{\circ} \mathrm{C}$ e $750^{\circ} \mathrm{C}$ todos com duração de 8 horas e resfriados ao ar.

Os ensaios de tração foram realizados em um simulador termomecânico Gleeble ${ }^{\circledR}$ modelo 3800 , instalado no Laboratório Nacional de Nanotecnologia (LNNano) e modelo 3500, instalado na linha XTMS no Laboratório Nacional de Luz Síncrotron (LNLS), ambos localizados no Centro Nacional de Pesquisa em Energia e Materiais (CNPEM). A tensão mecânica foi aplicada ao longo da direção de laminação com uma taxa de deformação de $10^{-4} \mathrm{~s}^{-1}$.

O procedimento de preparação das amostras para a análise de microscopia, envolveu as etapas de lixamento, com lixas de granulometria variando de I80 a I 200 mesh, polimento com alumina de $I \mu$ e ataque químico por imersão com Behara modificado $\left(\mathrm{HCl}+\mathrm{H}_{2} \mathrm{O}+\right.$ metabissulfito de potássio) por 15 minutos. As imagens foram obtidas utilizando um microscópio óptico digital Nikon Eclipse MA200.

\section{RESULTADOS E DISCUSSÃO}

As amostras nas condições como recebida (CR) e tratadas termicamente a $475^{\circ} \mathrm{C}(475), 600^{\circ} \mathrm{C}(600)$ e $750^{\circ} \mathrm{C}$ (750) foram ensaiadas na Gleeble ${ }^{\circledR} 3800$. A Figura la mostra as curvas tensão-deformação de engenharia obtidas nos ensaios de tração, e a partir dessas curvas, foi possível obter - limite de escoamento, limite de resistência à tração e alongamento \% total para cada condição analisada (Figura Ib).

A amostra tratada a $475^{\circ} \mathrm{C}$ apresentou o menor alongamento \% total, o que pode estar relacionado à precipitação de fases deletérias, especialmente a fase alfa linha $\left(\alpha^{\prime}\right)$, que é a principal causa do endurecimento, fragilização e perda de tenacidade dos AID nesta temperatura, conhecida como temperatura de fragilização. Além disso, essa fase

Tabela 2. Parâmetros de soldagem TIG para chapas do aço inoxidável duplex UNS S32304

\begin{tabular}{ccccccc}
\hline Corrente média & Corrente de base & Corrente de pico & Tensão Média & Velocidade & Tempo de pico & Tempo de base \\
\hline $143 \mathrm{~A}$ & $106 \mathrm{~A}$ & $180 \mathrm{~A}$ & $13 \mathrm{~V}$ & $37 \mathrm{~cm} / \mathrm{min}$ & $8,3 \mathrm{~ms}$ & $8,3 \mathrm{~ms}$ \\
\hline
\end{tabular}


também provoca a diminuição da resistência à corrosão do material $[2, \mathrm{II}, 15]$. As outras possíveis fases precipitadas são a austenita secundária $\left(\gamma_{2}\right)$ e nitreto de cromo $\left(\mathrm{Cr}_{2} \mathrm{~N}\right)$, que foram observadas por $[1,4,14,17,18]$.

$\mathrm{O}$ tratamento térmico a $600^{\circ} \mathrm{C}$ manteve a característica da curva tensão-deformação de engenharia e suas principais propriedades mecânicas bem próximas da amostra como recebida, o que pode ser justificado pela menor precipitação de fases deletérias, conforme investigado por outros autores $[1,11-13,19]$.

$\mathrm{O}$ tratamento térmico de $750^{\circ} \mathrm{C}$ mostrou valores maiores de limite de resistência à tração e alongamento \% total, entretanto, conforme reportado na literatura $[2,8,13,14,17-19]$ esta temperatura favorece a precipitação de fases deletérias como $\gamma_{2}, \mathrm{Cr}_{2} \mathrm{~N}$ e fase sigma $(\sigma)$, o que pode estar relacionado com as propriedades mecânicas obtidas.

Devido à similaridade das propriedades da amostra tratada a $600^{\circ} \mathrm{C}$ em relação à amostra como recebida, o que é desejável por manter as propriedades originais do material, e do ganho das propriedades obtido com o tratamento de $750^{\circ} \mathrm{C}$ decidiu-se fazer uma investigação comparativa entra as amostras soldadas sem tratamento térmico (Solda) e com tratamento térmico a $600^{\circ} \mathrm{C}$ (Solda +600 ) e a $750^{\circ} \mathrm{C}$ (Solda +750 ). As curvas tensão-deformação de engenharia para essas condições foram obtidas na Gleeble ${ }^{\circledR} 3500$ e estão expostas na Figura 2a, enquanto a Figura $2 \mathrm{~b}$ mostra as principais propriedades mecânicas obtidas a partir destas curvas.

$\mathrm{O}$ tratamento térmico a $600^{\circ} \mathrm{C}$ para amostra soldada promoveu melhora no limite de resistência à tração e alongamento \% total em relação à amostra soldada sem tratamento térmico. Para o limite de escoamento a redução foi inferior a $1 \%$ e está dentro da barra de erros. O tratamento térmico a $750^{\circ} \mathrm{C}$ para a amostra soldada propiciou um aumento no valor da ductilidade, conforme aconteceu para as amostras apenas tratadas nesta mesma temperatura, contudo causou perda na resistência mecânica.

Um estudo realizado sobre tensões residuais [20] para este mesmo material, sob mesma condição de soldagem, comprovou que o tratamento térmico a $600^{\circ} \mathrm{C}$ promoveu o alívio de tensões residuais, sem a precipitação de fases intermetálicas.

A partir da análise de microscopia óptica (Figura 3), foi possível avaliar qualitativamente o aumento do teor da fase austenítica (fase mais clara) com aumento da temperatura. Quando comparada às amostras apenas soldada e soldada com subsequente tratamento térmico a $600^{\circ} \mathrm{C}$, percebe-se uma pequena variação desta fase. Contudo, para a amostra soldada com subsequente tratamento térmico a $750^{\circ} \mathrm{C}$, o aumento da fase austenítica fica explícito, o que pode justificar o aumento da ductilidade. Já que a austenita tem
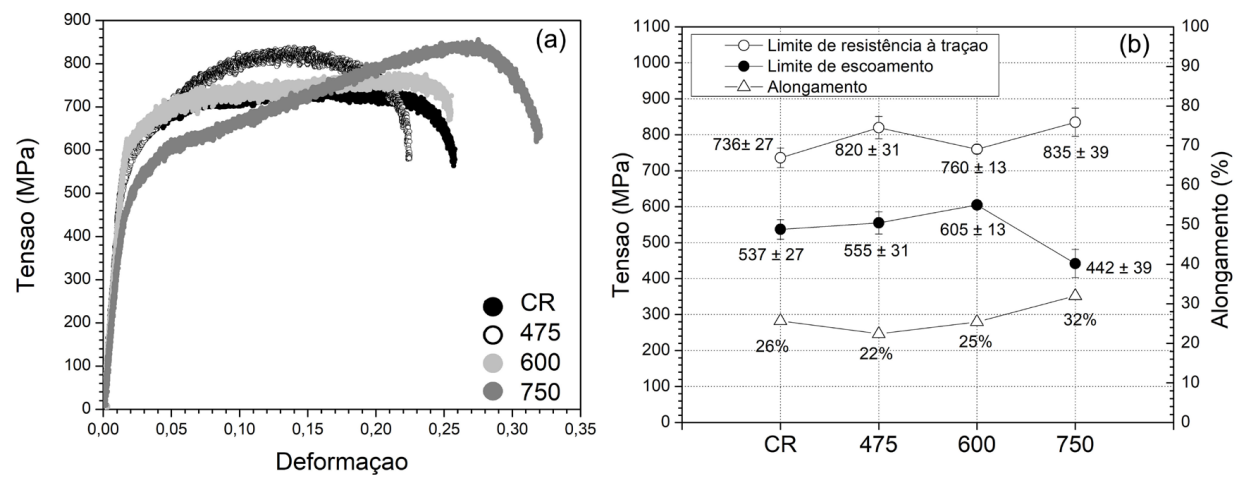

Figura I. (a) Curvas tensão-deformação de engenharia e (b) correlação das principais propriedades mecânicas das amostras do AID UNS S32304 nas condições como recebida (CR) e tratadas termicamente a 475,600 e $750^{\circ} \mathrm{C}$.
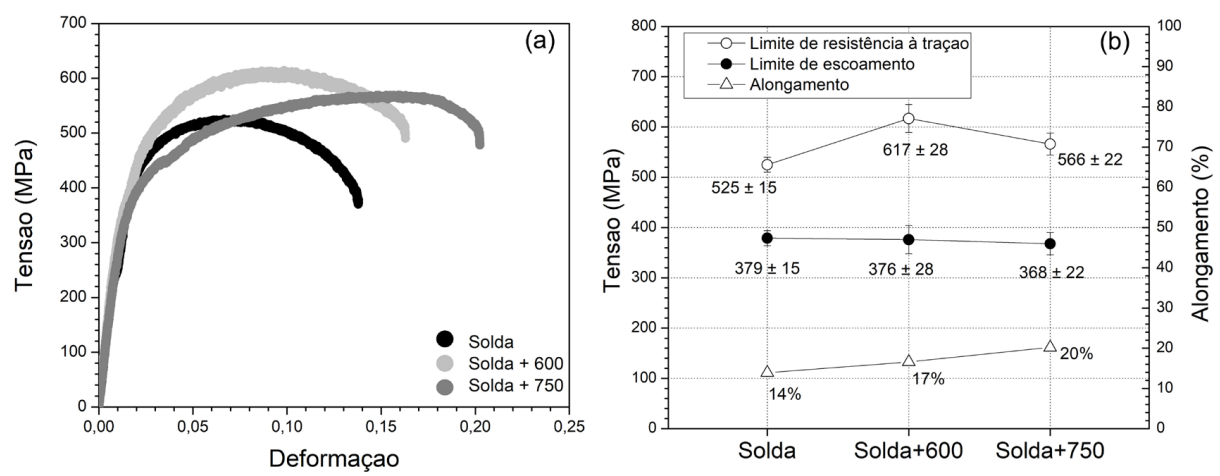

Figura 2. (a) Curvas tensão-deformação de engenharia e (b) correlação das principais propriedades mecânicas das amostras do AID UNS S32304 nas condições soldada (Solda), e soldadas com posterior tratamento térmico a $600^{\circ} \mathrm{C}$ (Solda +600 ) e a $750^{\circ} \mathrm{C}($ Solda +750$)$.

Tecnol. Metal. Mater. Miner., São Paulo, 



Figura 3. Microestrutura da zona fundida das amostras do AID UNS S32304 nas condições soldada (Solda), soldada com posterior tratamento térmico a $600^{\circ} \mathrm{C}($ Solda +600$)$ e a $750^{\circ} \mathrm{C}$ (Solda +750$)$. Região escura ferrita, região clara austenita. Ataque Behara modificado. Microscopia óptica, 100x.
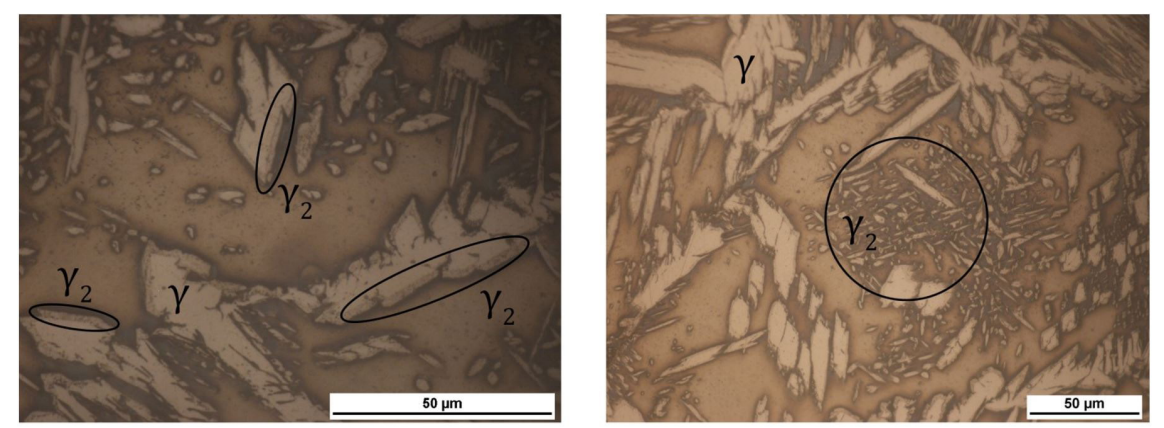

Figura 4. Microestrutura da zona fundida da amostra soldada com subsequente tratamento térmico a $750^{\circ} \mathrm{C}$, com destaque para as diferentes morfologias de austenita secundária. Ataque Behara modificado. Microscopia óptica, 1000x e 500x.

estrutura cristalina CFC, estrutura com maior quantidade de planos efetivos de deslizamentos, e maior liberdade para movimentação de discordâncias, portanto apresenta maior capacidade de suportar deformação plástica [2I].

Também foi constatada a presença de austenita secundária $\left(\gamma_{2}\right)$ na amostra soldada tratada termicamente a $750^{\circ} \mathrm{C}$ (Figura 4), formadas por dois mecanismos diferentes: associada à precipitação de nitretos formados anteriormente e que cresceram a partir da austenita pré existente [15]. Essa fase, apesar de contribuir para o aumento na ductilidade, é empobrecida em $\mathrm{Cr}$ quando comparada à matriz ferrítica e à austenita primária, tornando essas áreas suscetíveis à corrosão por pite [I].

\section{CONCLUSÃO}

Este estudo comprovou que o tratamento térmico a $600^{\circ} \mathrm{C}$ por $8 \mathrm{~h}$ seguido de resfriamento ao ar manteve as características do material similares à amostra na condição como recebida.
Para as amostras soldadas, o tratamento térmico a $600^{\circ} \mathrm{C}$ por $8 \mathrm{~h}$ seguido de resfriamento ao ar indicou ser o mais adequado tendo em vista o benefício obtido no alívio de tensões residuais, reportado na literatura, bem como o ganho na resistência mecânica e alongamento $\%$ total quando comparado às amostras soldadas sem tratamento térmico.

O tratamento térmico a $750^{\circ} \mathrm{C}$ por $8 \mathrm{~h}$ seguido de resfriamento ao ar para a amostra soldada propiciou o aumento no valor da ductilidade, contudo promoveu a redução da resistência mecânica, justificados pelo aumento da fração de austenita.

\section{Agradecimentos}

Os autores agradecem a CAPES Pró-Estratégia 2224/2012 e ao CNPQ pela concessão de bolsas de pesquisa e ao LNNano (proposta: STC 22766), LNLS (proposta: XTMS 20 I70659) e ao CNPEM pelo apoio na utilização das Gleeble ${ }^{\circledR}$.

\section{REFERÊNCIAS}

I Charles J. Duplex stainless steels, a review after DSS'07 held in Grado. Revue de Métallurgie. 2008; I05(3): I55- I7I.

2 Gunn RN. Duplex stainless steel: microstructure, properties and application. Abington: Woodhead Publishing Ltd; 1997.

3 Lippold JC, Kotecki DJ. Welding metallurgy and weldability of stainless steel. Hoboken: John Wiley \& Sons; 2005. 
4 Sahu JK, Krupp U, Ghosh RN, Chrit H-J. Effect of $475^{\circ} \mathrm{C}$ embrittlement on the mechanical properties of duplex stainless steel. Materials Science and Engineering A. 2009;508: I- I 4.

5 Hwang $\mathrm{H}$, Park Y. Effects of heat treatment on the phase ratio and corrosion resistance of duplex stainless steel. Materials Transactions. 2009;50:1548-1552.

$6 \mathrm{Ha} \mathrm{H}-\mathrm{Y}$, Jang $\mathrm{M}-\mathrm{H}$, Lee T-H, Moon J. Interpretation of the relation between ferrite fraction and pitting corrosion resistance of commercial 2205 duplex stainless steel. Corrosion Science. 20 I4;89:154-162.

7 Fonseca G, Barbosa LOR, Ferreira EA, Xavier CR, Castro JA. Microstructural, mechanical, and electrochemical analysis of duplex and superduplex stainless steels welded with the autogenous TIG process using different heat input. Metals. 2017;7(I2):538-558.

8 Dos Santos DC, Magnabosco R. Kinetic study to predict sigma phase formation in duplex stainless steels. Metallurgical and Materials Transactions. A, Physical Metallurgy and Materials Science. 2016;47(4):I554-I565.

9 Tumu DP, Subramani P, Kumar KG, Manikandan MB, Mohan CG, Arivazhagan N, Rajan DN. Investigation on microstructure and tensile properties of dissimilar weld joints between AISI 3 I6I and duplex 2205 stainless steel. IOP Conference Series. Materials Science and Engineering. 2018;402(I):I-7.

10 Betini EG, Gomes MP, Milagre MX, Machado CSC, Reis LAM, Mucsi CS, Orlando MTD, Luz TS, Martinez LG, Rossi JL. Study on welding thermal cycle and residual stress of UNS S32304 duplex stainless steel selected as external shield for a transport packaging of Mo-99. Brazilian Journal of Radiation Sciences. 2019;7(2A): I-I3.

I I International Molybdenum Association. Practical guidelines for the fabrication of duplex stainless steels. London: IMOA; 2014.

12 Pohl M, Storz O, Glogowski T. Effect of intermetallic precipitations on the properties of duplex stainless steel. Materials Characterization. 2007;58:65-7I.

13 Nilsson JO. Super duplex stainless steels. Materials Science and Technology. 1992;8:685-700.

14 Londoño AJR. Precipitação de fases intermetálicas e austenita secundária na ZAC de soldagens multipasse de aços inoxidáveis duplex [tese]. São Paulo: Escola Politécnica da Universidade de São Paulo; 2001.

15 Ramirez AJ, Lippold JC, Brandi SD. The relationship between chromium nitride and secondary austenite precipitation in duplex stainless steels. Metallurgical and Materials Transactions. A, Physical Metallurgy and Materials Science. 2003;34A:1575-1597.

16 Marques PV, Modenesi PJ, Bracarense AQ. Soldagem. Fundamentos e tecnologia. 3. ed. Belo Horizonte: Editora UFMG; 2011.

17 Machado CSC. Perfis de tensão residual do aço duplex UNS S32304 submetido a diferentes processos de soldagem TIG e condições de tratamento térmico [dissertação]. Vitória: Universidade Federal do Espírito Santo; 2015.

18 Milagre MX. Perfis de tensão residual do aço duplex UNS S32304 submetido a processo de soldagem TIG e diferentes condições de tratamento térmico de têmpera [dissertação]. Vitória: Universidade Federal do Espírito Santo; 2015.

19 Calliari I, Brunelli K, Zanellato M, Ramous E, Bertelli R. Microstructural modification during isothermal ageing of a low nickel duplex stainless steel. Journal of Materials Science. 2009;44:3764-3769.

20 Milagre MX, Machado CSC, Orlando MTD, Rossi JL, Martinez LG, Macêdo MCS, Scandian C. Efeito do tratamento térmico na magnitude das tensões residuais do aço inoxidável duplex UNS S32304. In: Grupo de Física Aplicada/ UFES. Anais do $4^{\circ}$ Encontro Científico de Física Aplicada; 20I4; Vitória, Brasil. Blucher Proceedings; 2014. p. II8-I2I.

2I Reed-Hill RE. Physical Metallurgy Principles. 2. ed. New York: Van Nostrand: Litton Educational Publishing; 1973.

Recebido em: 30 Nov. 2018

Aceito em: 13 Out. 2019 